Interdisciplinary Analyses of Social-Ecological Systems

\title{
Impact of Natural Disasters, Psychological and Physical Effects on the Population
}

\author{
Aliona Tihon \\ "Nicolae Testemitanu" State University of Medicine and the Pharmacy Chisinau, Republic of Moldova
} doi: https://doi.org/10.21467/abstracts.93.73

\begin{abstract}
A natural hazard is a geophysical, atmospheric or hydrological event (e.g., earthquake, landslide, tsunami, windstorm, flood or drought) that has the potential to cause harm or loss, while a natural disaster is the occurrence of an extremely hazardous event that impacts on communities causing damage, disruption, and casualties, and leaving the affected communities unable to function normally without outside assistance. The definition of natural disaster impact (NDI) can change according to both the aim of the study and the scientist assessing it. It can be defined as constituting the direct, indirect and intangible losses caused by the environment and society by a natural disaster. Direct losses include physical effects such as destruction and changes that reduce the functionality of an individual or structure. Damages to people (death/injury), buildings, their contents, and vehicles are included, as are clean-up and disposal costs. Indirect losses affect society by disrupting or damaging utility services and local businesses. Loss of revenue; increase in cost; expenses connected to the provision of assistance, lodging, and drinking water; and costs associated with the need to drive long distances because of blocked roads are included. Intangible losses include psychological impairments caused by both direct and intangible losses that individuals personally suffer during the disaster.

The Natural Disaster Impact Assessment (NDIA) is crucial in helping individuals to estimate replacement costs and to conduct cost-benefit analyses in allotting resources to prevent and mitigate the consequences of damage. A general NDIA procedure has not yet been developed; several approaches are available in the literature and their applicability depend on the accessibility of damage data.

Psychological effects. According to the Conservation Resource Model, people try to protect resources such as objects (housing, possessions, etc.), social roles (employment, marriages, etc.), energy (time and monetary investments), and personal characteristics (e.g., self-confidence). The threatened or actual loss of these resources as caused by a natural disaster leads to psychological distress. Frequently observed conditions such as minor emotional disorders seldom come to the attention of psychiatrists but may negatively affect social relationships and work performance. Commonly detected symptoms are fatigue, tics, and cognitive experiences such as confusion, impaired concentration, and attention deficit disorder. Emotional signs such as anxiety, depression, and grief, as well as behavioral effects such as sleep and appetite changes and substance abuse, were also reported. Even effects on suicide rates were detected: earthquake victims (people who had lost family members residing with them, were injured, or experienced property loss) were 1.46 times more likely than non-victims to commit suicide. All these effects can be mild and transitory or can lead to Post Traumatic Stress Disorder (PTSD). The mental states of victims can include three stages:

$>$ an immediate reaction involving distressing symptoms accompanying adaptive stress;

$>$ the post-immediate phase, which includes symptoms of maladaptive stress (confusion, agitation, and occasionally neurotic or psychotic reactions);

$>$ the long-term sequel phase, which involves a return to normal health or the onset of PTSD, which can sometimes yield a chronic phase that involves personality changes.
\end{abstract}


The Second Eurasian RISK-2020 Conference and Symposium

These surveys make it possible to monitor the most fragile segments of the population, including people with pre-existing mental illness, racial and ethnic minorities, and children, in which symptoms may differ depending on age. Gender differences arise as well: for instance, after an earthquake, women report greater emotional distress and mental health problems than do men, but the occurrence of addiction disorders among women is much lower.

Physical effects encompass symptoms affecting people who have not been directly involved in a disaster. The deterioration of hygiene, housing, and basic services can induce the outbreak of diseases such as leptospirosis or increase the risk of morbidity and mortality caused by communicable diseases. In developing countries, for instance, contagious and non-contagious diseases are reported during the first weeks after floods. Moreover, in some environments, even the incidence of snake bites can increase. Disaster-related stress can have several secondary impacts on human health, such as effects on the human immune system, diabetes, and gastroduodenal ulcer In addition, after major earthquakes, the number of patients with Acute Myocardial Infarction (AMI) has been reported to increase 3.5-fold, and the part of women with AMI seems significantly greater than in the years preceding the disaster. The numerous experiences of damage assessment performed in different economic frameworks show that developing countries are more strongly hit than developed ones: as economies develop, there are fewer disaster-related deaths and damages/GDP. Nevertheless, increasing wealth causes relatively higher losses in high-income nations. Increases in income increase the private demand for safety; higher-income enables countries to employ additional, costly precautionary measures. Yet, in countries with a concentration of assets that is larger than the countermeasures, the income vulnerability relationship can be inverted, especially in the case of disasters related to behavioral choices such as floods and landslides. Two major constraints obstruct the assessment of the impact of disasters: the first is the lack of shared assessment methodologies. On the other hand, conveying all the different assessments in a single methodological approach is objectively an extremely complicated task that can be handled exclusively by multidisciplinary staff, having all the skills to cope with a multifaceted task as disaster impact assessment.

The second problem is related to data availability. It is impossible to decide a priori the most opportune time to gather data and to undertake impact assessment, as it will depend on the type of phenomenon causing the disaster, its magnitude, and the scope of the assessment. In addition, continuous data gathering also once the emergency phase has passed ensures detection of long-term effects, as either economic impacts or psychological consequences on people affected. On the contrary, dealing with the impact of phenomena occurred in the past, data gathering becomes very complicated: both the amount of data available and their level of detail can below and cannot be significantly increased, even by further research. In these cases, simplified approaches can be used to perform relative assessments based on a minimum amount of information. These approaches aim to supply quantitative indices expressing the impact of different disasters in order to make them comparable even if they occurred both at different times and in different areas. Specifically, for landslides, a structured approach aiming to collect data and transforming them into relative damage indices is presented. This approach can be used for a) current events for which monetary assessments are not (or not yet) available, and B) past landslides for which monetary assessments of costs are quite impossible to obtain. In both of these situations, the approach focuses on the minimum amount of information that can be used to define the impact caused by different landslides in order to perform impact comparisons. It must be understood when using this approach that one is dealing with relative assessments and their reliability strictly depends on the reliability of the data employed. 